\title{
TRAINING TO IMRPOVE COLLISION DETECTION IN OLDER ADULTS
}

\author{
Carissa M. Lemon, Denton DeLoss, \& George J. Andersen \\ University of California, Riverside \\ Riverside, California, USA \\ Email: clemo001@ucr.edu
}

\begin{abstract}
Summary: Previous studies have indicated a decline in the ability of older adults to detect impending collisions. In addition, previous research has demonstrated that collision detection performance of college-aged participants can be improved with perceptual learning. The present study examined whether perceptual learning can improve performance of older participants on a collision detection task $(\mathrm{N}=12)$. The experiment was conducted over seven days with each day consisting of a 1-hr session. Thresholds for three observer speeds were measured prior to training using a two-alternative forced choice procedure during which participants indicated whether an approaching object would result in a collision or noncollision event. Participants were then trained near threshold at one of these speeds for 5 days. After training participants' thresholds were measured again. Results indicate a significant reduction in the time needed to detect a collision for the trained condition as well as an untrained observer speed condition. Results demonstrate that collision detection performance for older participants can be improved with perceptual learning and may transfer to untrained observer speed.
\end{abstract}

\section{BACKGROUND}

The number of older drivers in the United States is in the midst of doubling and by 2030 one in five adults will be 65 and older (Ortman, Velkoff, \& Hogan, 2014). These population statistics are noteworthy because accident risk has been shown to increase with age-with accident risk nearly tripling from middle to late adulthood (Harris, 1999). Further, when in a crash, older drivers are more often at fault and have been shown to have difficulties when making complex driving maneuvers (NHTSA, 2010). While some of these accidents can be attributed to changes of the eye caused by normal aging (e.g. changes in the lens or pupil size) many older adults experience declines in perceptual and cognitive abilities, making them less safe drivers (Kline et al., 1992).

One perceptual ability that may account to the age-related deviations in driving skill is the decline of motion perception. Older adults have been shown to have deficits in determining the direction of coherent motion and discriminating noise from global motion (Trick \& Silverman, 1992; Bennett, Sekuler, \& Sekuler, 2007). More complex motion tasks show declines as well, such as the perception of speed and detection of an imminent collision (Enriquez \& Andersen, 2006). These findings are supported by research that demonstrates that older adults are less accurate at judging whether a collision will occur as compared to younger adults (DeLucia, Bleckley, Meyer, \& Bush, 2003). Additionally, older adults report that collisions will occur sooner than simulated (Schiff, Oldak, \& Shah, 1992), which is likely a compensatory response.

Research that has focused specifically on collision detection has indicated that older adults are less sensitive at detecting collisions, particularly at high speeds. Sensitivity has been shown to decrease when observer motion is present and when the speed of the observer is increased, 
particularly at speeds greater than $45 \mathrm{mph}$ (Enriquez \& Andersen, 2006). These results suggest that increased accident rates for older drivers may be due to a decline in the ability to detect collisions at high speeds.

One way to mitigate these deficits in collision detection is to implement a perceptual learning (PL) paradigm. PL is defined as the long term improvement in performance on a perceptual task following training. Given that the visual system has been found to maintain plasticity into adulthood (Kempermann, Gast, \& Gage, 2002; Sasaki, Nanez, \& Watanabe, 2010) PL may be a useful intervention for improving age related declines in vision and recent research suggests that PL may be effective for older adults. Various perceptual tasks have been found to improve older adults performance after training including contrast sensitivity (DeLoss, Watanabe, \& Andersen, 2015), texture discrimination (Andersen, Ni, Bower, \& Watanabe, 2010), and motion discrimination (Bower \& Andersen, 2012; Ball \& Sekuler, 1986). This begs the question: is PL effective at training higher levels of processing in older adults and does this improvement transfer to other stimuli?

For example, Andersen et al. (2010) found that 5 days of training with near threshold conditions in a texture discrimination task resulted in improved threshold for older adults that were similar to thresholds for untrained younger adults. In addition, Yotsomoto, Wantanabe, and Sasaki (2008) found that PL training with a texture discrimination task resulted in increased white matter in V1 for older adults but not for younger adults. This finding indicated that the visual system maintains plasticity into advanced age.

Previous studies of PL have examined whether these improvements transfer to untrained stimuli or tasks. For example, improvements have been found to transfer to untrained orientations (Karni \& Sagi, 1991) and locations ((Schoups, Vogels, \& Orban, 1995). Likelihood of transfer, or specificity, has been found to depend on the difficulty of the task (Ahissar \& Hochstein, 1997). Thus, examining specificity is important to counteract age-related declines in vision and the present study seeks to assess this specificity.

A previous study has examined training and transfer of a high level of processing task for college-aged participants (DeLoss, Bian, Watanabe, \& Andersen, 2015). Testing and training was performed over 7 sessions on a collision detection task. Participants were trained near threshold at one observer speed for 5 days. After training, participants were tested and showed a significant reduction in the time needed to detect a collision at the trained speed. This improvement was also found to transfer to the higher observer speed condition. A second experiment was conducted to determine whether this improvement was due to training near threshold or whether this improvement was merely due to practice with the task. Training with stimuli above threshold showed no significant improvement in performance. Therefore, the purpose of this study was to determine if training higher levels of processing was effective for older adults and to examine if improvement transferred. Older adults were tested and trained on a collision detection task for 7days. Duration threshold, the amount of time needed to determine a collision event, was recorded. 


\section{METHODS}

\section{Participants}

Twelve older participants $(M=74.4, S D=5.05)$ from the University of California, Riverside (six males and six females) participated in the experiment. All participants had normal or correctedto-normal vision and were asked to wear appropriate corrective lenses (see Table 1). Participants were unaware of the purposes of the study and were reimbursed $\$ 15$ per hour.

\section{Apparatus}

Stimuli were generated using software written using MATLAB (The Mathworks inc., version 2015b) and the Psychophysics Toolbox (Brainard, 1997; Pelli, 1997). The stimuli were presented on a 58-inch plasma display (Panasonic TH-58PF12UK). The display had a refresh rate of $60 \mathrm{~Hz}$ and a resolution of 1920 X 1080. A Dell precision T7500 equipped with dual Intel Xeon E5506 processors using Window 7 (Service Pack 1) operating system equipped with an NVIDIA Quadro FX 4800 graphics card was used.

\section{Procedure and Stimuli}

The stimuli consisted of 1.5 hours per day of testing or training over 7 days within the span of 3 weeks. The stimuli were viewed binocularly with the monitor at a distance of $60 \mathrm{~cm}$. Participants that had corrected vision were asked to wear their corrective lenses. Participants were presented with a 3-D scene that consisted of a randomly generated texture. Such a texture was chosen to prevent participants from using features within the texture as references to determine the object's motion. The viewpoint was $1.2 \mathrm{~m}$ above the ground plane

On each trial forward motion was simulated. This sphere translated toward the observer with the approaching spherical object was initially positioned to the left or right of the roadway along an arc (approx. $20^{\circ}$ from the center of the display) at a fixed distance 115 units from the viewpoint. The object moved on a linear trajectory. Half of the trials consisted of motion trajectories that would collide with the observer and the other half of trials would pass by the observer. Collision events were created by crossing the trajectory, from the initial position, with the viewpoint of the observer.

Three speed conditions were used. In one condition the observer travelled at $100 \mathrm{~km} / \mathrm{h}$ and the sphere approached at $20 \mathrm{~km} / \mathrm{h}$. In the second condition the observer travelled at $60 \mathrm{~km} / \mathrm{h}$ and the sphere approached at $60 \mathrm{~km} / \mathrm{h}$. In the third condition the observer travelled at $20 \mathrm{~km} / \mathrm{h}$ and the sphere approached at $100 \mathrm{~km} / \mathrm{h}$. These speeds were chosen in order to maintain a constant relative speed and time to contact (TTC) across the three conditions.

Task practice. On day 1 of the experiment participants were provided with 4 trials to become familiar with the stimuli. Following these trials participants were given 10 practice trials on which they had to correctly identify 9 out of 10 trials to continue on to the experimental trials. Participants were instructed to enter ' 4 ' on the number keypad to indicate that they perceived an impending collision and ' 6 ' to indicate they perceived a non-collision.

Testing. Days 1 and 7 were testing days. Participants' duration thresholds for all three speed conditions were tested on these days. On days 1 and 7, pretraining TTC thresholds for the three 
speed levels were assessed using QUEST (Watson \& Pelli, 1983). QUEST is an adaptive psychometric procedure that relies on Bayesian methods to estimate threshold. QUEST was initialized with a criterion of $75 \%$ correct, $\beta=1.6, \delta=0.01, \gamma=0.5$. On testing days the duration threshold for QUEST was set to 7000ms. For each speed level participants completed 100 trials with conditions speed randomized.

Training. Days 2 through 6 were training days. Participants completed 300 trials of $60 \mathrm{~km} / \mathrm{h}$ object and $60 \mathrm{~km} / \mathrm{h}$ observer speeds. Quest was initialized with the same criterion, beta, delta and gamma as the testing days. Duration thresholds were based on the $75 \%$ correct threshold from the prior day's performance. This was done to account for any improvement in performance. In total training consisted of 1,500 trials. Auditory feedback was given.

\section{RESULTS}

In order to test for differences in duration threshold before and after training, a training (pretraining, post-training) by object/observer speed $(20 / 100 \mathrm{~km} / \mathrm{h}, 60 / 60 \mathrm{~km} / \mathrm{h}, 100 / 20 \mathrm{~km} / \mathrm{h}$ ) repeated measures analysis of variance was performed. A significant main effect of day was found for training $F(1,11)=16.277, p=0.002, \eta^{2}=.597$. This indicates that participants' duration threshold decreased after training (see Figure 1). The main effect of speed was not significant with object speed $F(2,22)=1.792, p>.05, \eta^{2}=.150$.

To quantify the amount of transfer at different speed levels (see Figure 2), planned comparisons were used to compare object speed to performance from pretraining to post-training.

Comparisons from pretraining $(M=7.922)$ to post-training $(M=7.324)$ at the first object/observer speed $(20 / 100 \mathrm{~km} / \mathrm{h})$ was significant $t(11)=2.77, p=0.018, d=0.802$. For the trained

object/observer speed condition $(60 / 60 \mathrm{~km} / \mathrm{h})$ a comparison of pretraining $(M=7.674)$ with posttraining $(M=7.117)$ was significant $t(11)=4.214, p=0.001, d=1.204$. Finally, the last untrained object/observer speed condition $(100 / 20 \mathrm{~km} / \mathrm{h})$ was not significant from pretraining to posttraining with $t(11)=1.294, p=0.222, d=0.373$. This suggests that training was effective for the trained object speed and the slow object speed condition. These statistical tests demonstrate that training with a collision detection task over 5 days was effective. While an interaction between training and object speed was not significant, t-test show a significant decrease in duration threshold after training in an untrained condition.

\section{DISCUSSION}

In the present study, we examined the usefulness of training older adults using a higher order processing task, collision detection. The results of this study indicate that PL that is directed at higher levels of processing in older adults is effective. Further, this training appears to transfer to higher observer speeds, which is of importance because many older adults have been shown to have difficulty with higher speeds. As seen in Figure 2, a reduction of approximately 550 milliseconds was found for the trained object/observer speed condition of $60 / 60 \mathrm{~km} / \mathrm{h}$ and a reduction of 600 milliseconds was found for an untrained object/observer speed condition of $20 / 100 \mathrm{~km} / \mathrm{h}$, providing observers with more time to respond to a collision event.

Compared to results found by DeLoss et al. (2015) involving younger adults, older adults in this study required more time to respond to an impending collision. However, both younger and older adults show a similar learning trend, with significant improvement on the trained condition as 
well as on an untrained slower object speed condition, signifying that the training follows a similar trajectory. Failure to transfer to both untrained condition indicates that these results cannot be accounted for practice with the task.

Additionally, some PL studies have shown that the perceptual improvements are specific to the trained stimuli and do not transfer to other untrained stimuli features or tasks. Lack of transfer has been found for training on low-level stimuli such as contrast, orientation and texture (Karni $\&$ Sagi, 1991). However, one study examined the frequency of transfer on various perceptual tasks and concluded that likelihood of transfer was dependent on the complexity of the task (Fine \& Jacobs, 2002). Tasks involving simple stimuli and judgments along a single perceptual dimension tended to show only small amounts of learning and were less likely to transfer, whereas more complex stimuli showed greater magnitude of learning and were more likely to transfer. It is not surprising that transfer was found in this study given the complexity of the task. Future research will need to focus on determining why transfer did not occur for the $100 / 20 \mathrm{~km} / \mathrm{h}$ object/observer speed condition.

The speeds chosen for this study were chosen due to their applicability to real world driving scenarios. Older adults show declines in the ability to detect a collision, require more time to respond appropriately, and have greater difficulty when travelling at higher speeds. Results demonstrate a reduction in the time needed to detect a collision following training and this effect generalizes to a faster untrained observer speed condition, having implications for the mitigation of driving skill declines using a training paradigm.

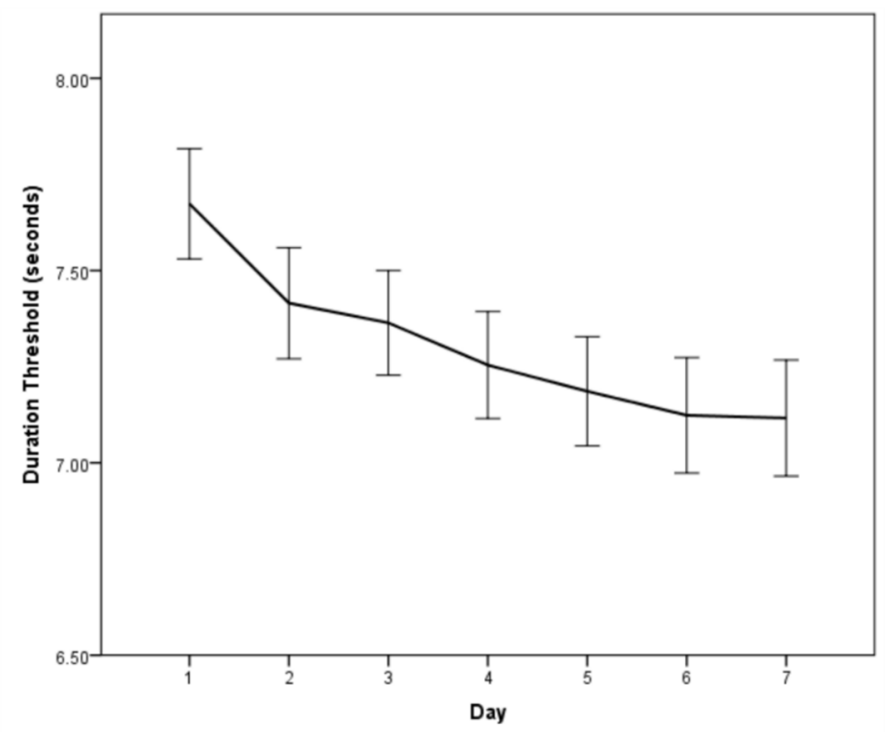

Figure 1. Duration over 7 days

Mean duration threshold at the trained object/observer speed of $60 \mathrm{~km} / \mathrm{h}$ as a function of day. 


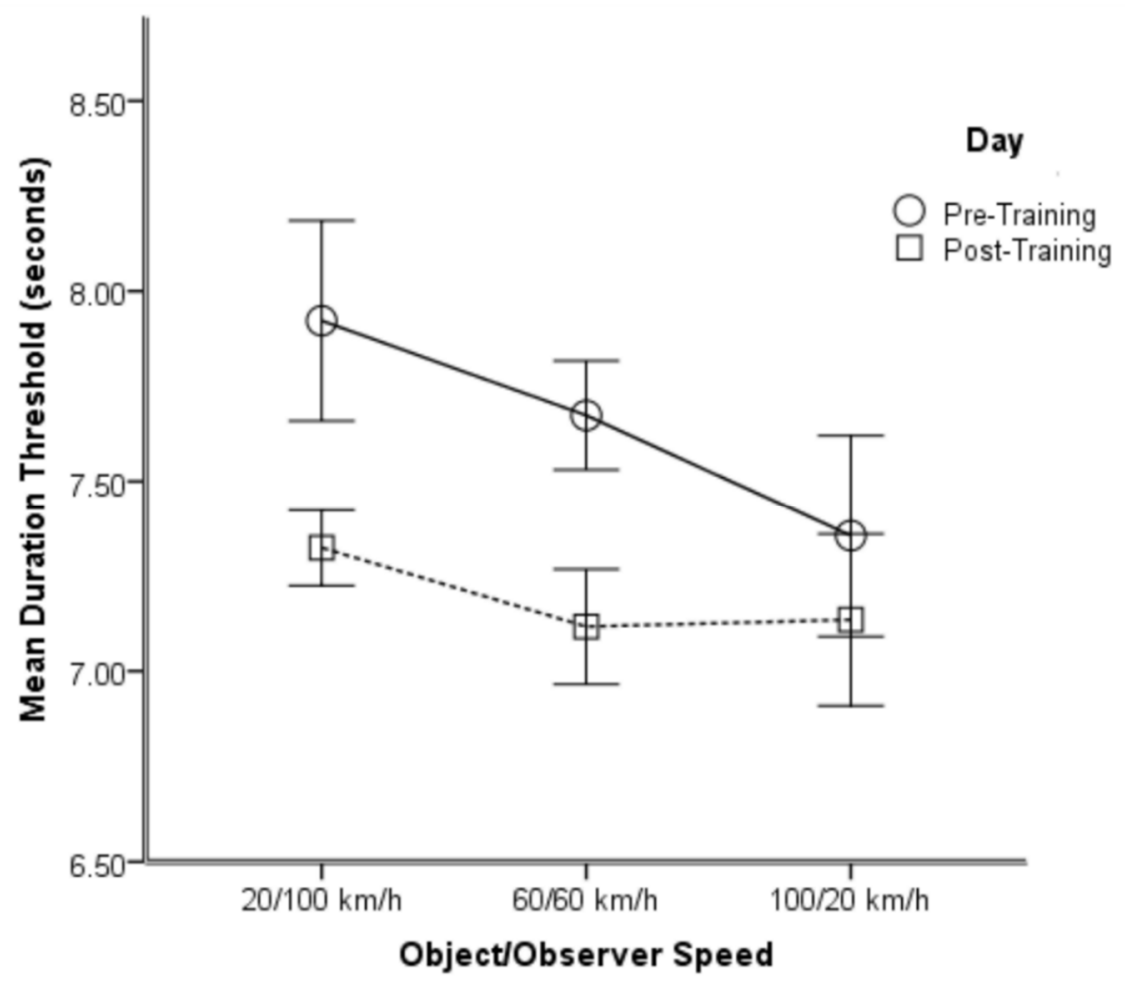

Figure 2. Duration as function of speed

Mean duration thresholds as a function of training (pre-training to post-training).

\section{ACKNOWLEDGMENT}

Research supported by AG031941

\section{REFERENCES}

Ahissar, M., \& Hochstein, S. (1997). Task difficulty and the specificity of perceptual learning. Nature, 387(6631), 401-406.

Andersen, G. J., \& Kim, R. D. (2001). Perceptual information and attentional constraints in visual search of collision events. Journal of Experimental Psychology: Human Perception and Performance, 27(5), 1039.

Andersen, G. J., \& Enriquez, A. (2006). Aging and the detection of observer and moving object collisions. Psychology and Aging, 21(1), 74-85.

Andersen, G. J., Ni, R., Bower, J. D., \& Watanabe, T. (2010). Perceptual learning, aging, and improved visual performance in early stages of visual processing. Journal of Vision, 10(13), 4-4.

Bower, J. D., \& Andersen, G. J. (2012). Aging, perceptual learning, and changes in efficiency of motion processing. Vision research, 61, 144-56.

Bennett, P. J., Sekuler, R., \& Sekuler, A. B. (2007). The effects of aging on motion detection and direction identification. Vision Research, 47(6), 799-809. 
DeLoss, D. J., Bian, Z., Watanabe, T., \& Andersen, G. J. (2015). Behavioral training to improve collision detection. Journal of Vision, 15(10), 2-2.

DeLoss, D. J., Watanabe, T., \& Andersen, G. J. (2015). Improving vision among older adults behavioral training to improve sight. Psychological Science, 26(4), 456-466.

DeLucia, P. R., Kaiser, M. K., Bush, J. M., Meyer, L. E., \& Sweet, B. T. (2003). Information integration in judgments of time to contact. Quarterly Journal of Experimental Psychology, $56 A, 1165-1189$.

Fine, I., \& Jacobs, R. A. (2002). Comparing perceptual learning across tasks: A review. Journal of Vision, 2(2), 5-5.

Fiorentini, A., \& Berardi, N. (1981). Learning in grating waveform discrimination: Specificity for orientation and spatial frequency. Vision Research, 21(7), 1149-1158.

Gilmore, G. C., Wenk, H. E., Naylor, L. A., \& Stuve, T. A. (1992). Motion perception and aging. Psychology and aging, 7(4), 654.

Karni, A., \& Sagi, D. (1991). Where practice makes perfect in texture discrimination: Evidence for primary visual cortex plasticity. Proceedings of the National Academy of Sciences, USA, 88(11), 4966-4970.

Kline, D. W., Kline, T. J., Fozard, J. L., Kosnik, W., Schieber, F., \& Sekuler, R. (1992). Vision, aging, and driving: The problems of older drivers. Journal of gerontology, 47(1), 27-34.

Ortman, J. M., Velkoff, V. A., \& Hogan, H. (2014). An aging nation: the older population in the United States. Washington, DC: US Census Bureau, 25-1140.

Sasaki, Y., Nanez, J. E., \& Watanabe, T. (2010). Advances in visual perceptual learning and plasticity. Nature Reviews Neuroscience, 11(1), 53-60.

Trick, G. L., \& Silverman, S. E. (1991). Visual sensitivity to motion; Age related changes and deficits in senile dementia of the Alzheimer type. Neurology, 41(9), 1437-1437.

Watson, A. B., \& Pelli, D. G. (1983). QUEST: A Bayesian adaptive psychometric method. Attention, Perception, \& Psychophysics, 33(2), 113-120.

Yotsumoto, Y., Watanabe, T., \& Sasaki, Y. (2008). Different dynamics of performance and brain activation in the time course of perceptual learning. Neuron, 57(6) 